\title{
Review of: "Stressful Events and Oral Health Related Quality of Life Aboard: a Longitudinal Study"
}

\author{
Nor Adinar Baharuddin ${ }^{1}$
}

1 Universiti Malaya

Potential competing interests: The author(s) declared that no potential competing interests exist.

This manuscript looked into how occupational stress could have an impact on the OHRQOL in the military population. It is challenging to plan for a longitudinal study and subsequently publish the findings. The objective was clear and methodology was well written. Nevertheless, here are few comments:

1. Be specific with inclusion criteria. It is already in the manuscript, just need to make it clear to readers.

2. Identify number (percentage) and reasons for dropped out. Deliberate in the discussion.

3. Table 1. What is the unit for OHIP-14 and PSS-14?

Definitely, this study will provide brilliant input to the authority as to how to improve the situation in the military population especially during training. Stress management would be one of the ways to go about it.

Accepted with minor corrections as mentioned above. Thanks 